\title{
Left-orderability and cyclic branched coverings
}

\author{
YING HU
}

\begin{abstract}
We provide an alternative proof of a sufficient condition for the fundamental group of the $n^{\text {th }}$ cyclic branched cover of $S^{3}$ along a prime knot $K$ to be left-orderable, which is originally due to Boyer, Gordon and Watson. As an application of this sufficient condition, we show that for any $(p, q)$ two-bridge knot, with $p \equiv 3 \bmod 4$, there are only finitely many cyclic branched covers whose fundamental groups are not left-orderable. This answers a question posed by Dąbkowski, Przytycki and Togha.
\end{abstract}

57M05; 57M12, 57M27

\section{Introduction}

\subsection{Background and results}

A group $G$ is called left-orderable if there exists a strict total ordering $<$ on the set of group elements, such that given any two elements $a$ and $b$ in $G$, if $a<b$ then $c a<c b$ for any $c \in G$.

It is known that any connected, compact, orientable 3-manifold with a positive first Betti number has a left-orderable fundamental group; see Boyer, Rolfsen and Wiest [4, Theorem 1.1] and Howie and Short [12]. In contrast, for a rational homology sphere, the left-orderability of its fundamental group is a nontrivial property, which is closely related to the co-oriented taut foliations on the manifold; see Calegari and Dunfield [5]. Moreover, Boyer, Gordon and Watson [3] conjectured that an irreducible rational homology 3-sphere $M$ is an L-space (see Ozsváth and Szabó [19]) if and only if its fundamental group $\pi_{1}(M)$ is not left-orderable.

Let $X_{K}$ be the complementary space obtained by removing an open tubular neighborhood of the knot $K$ from the three sphere $S^{3}$ and $X_{K}^{(n)}$ be the $n^{\text {th }}$ cyclic branched cover of $S^{3}$ branched over the knot $K$. The first Betti number $b_{1}\left(X_{K}^{(n)}\right)$ equals zero if and only if no root of the Alexander polynomial $\Delta_{K}(t)$ is an $n^{\text {th }}$ root of unity. Hence, most of the cyclic branched covers along a knot are rational homology spheres. In particular, this is the case if $n$ is a prime power. 
For this class of rational homology spheres, the L-space conjecture [3] has been verified in the following cases, where they are all L-spaces and have non-left-orderable fundamental groups:

- The twofold branched cover of any nonsplit alternating link; see Boyer, Gordon and Watson [3], Greene [10], Ito [13] and Ozsváth and Szabó [20].

- The $n^{\text {th }}$ cyclic branched cover of a $(p, q)$ two-bridge knot with $p / q=2 m+\frac{1}{2 k}$, $m k>0$ and $n$ arbitrary; see Da̧bkowski and Przytycki [7], and Peters [21].

- The $3^{\text {rd }}$ and $4^{\text {th }}$ cyclic branched cover of a $(p, q)$ two-bridge knot with

$$
p / q=n_{1}+\frac{1}{1+\frac{1}{n_{2}}},
$$

where $n_{1}, n_{2}$ are positive odd integers (ie $p / q=2 m+\frac{1}{2 k}, m k<0$ ); see Dạbkowski and Przytycki [7], Gordon and Lidman [9], Peters [21] and Teragaito [25].

The motivation of this paper is a question posed in [7]: Given a two-bridge knot $K$, is $\pi_{1}\left(X_{K}^{(n)}\right)$ always non-left-orderable if $b_{1}\left(X_{K}^{(n)}\right)=0$ ? We answer this question negatively. In fact, we prove that for $(p, q)$ two-bridge knots with $p \equiv 3 \bmod 4$, there are only finitely many cyclic branched covers that have non-left-orderable fundamental groups. At the end, we will present the knot $5_{2}$ as an example and show that the fundamental group $\pi_{1}\left(X_{5_{2}}^{(n)}\right)$ is left-orderable if $n \geq 9$. Shortly after this article was posted on the arXiv, Tran [26] computed an upper bound (depending on the knot) on the order $n$ so that the $n^{\text {th }}$ cyclic branched cover has a non-left-orderable fundamental group for a large class of two-bridge knots.

A similar question for hyperbolic knots was also posed in [7] and was first answered by Clay, Lidman and Watson [6, Proposition 23]. They showed that the twofold branched cover of $S^{3}$ along the Conway knot, which is a nonalternating hyperbolic knot listed as $11 \mathrm{n} 34$ in the standard knot tables, has a left-orderable fundamental group, and so do all even order cyclic branched covers.

\subsection{Plan of the paper}

Section 2 is devoted to proving Lemma 2.1, essential to our proof of Theorem 3.1.

Lemma 2.1 Given a knot $K$ in $S^{3}$, denote by $Z$ a meridional element in the knot group $\pi_{1}\left(X_{K}\right)$. Suppose that there exists a group homomorphism $\rho$ from $\pi_{1}\left(X_{K}\right)$ to a group $G$ and $\rho\left(Z^{n}\right)$ is in the center of $G$. Then $\rho$ induces a group homomorphism from $\pi_{1}\left(X_{K}^{(n)}\right)$ to $G$. In particular, if $\rho$ is nonabelian, then the induced homomorphism is nontrivial. 
We finish the proof of Theorem 3.1 in Section 3.

Theorem 3.1 Given any prime knot $K$ in $S^{3}$, denote by $Z$ a meridional element of $\pi_{1}\left(X_{K}\right)$. If there exists a nonabelian representation $\pi_{1}\left(X_{K}\right)$ to $\operatorname{SL}(2, \mathbb{R})$ such that $Z^{n}$ is sent to $\pm I$ then the fundamental group $\pi_{1}\left(X_{K}^{(n)}\right)$ is left-orderable.

This result was first observed by Boyer, Gordon and Watson:

Theorem [3, Theorem 6] Let $K$ be a prime knot in the 3-sphere and suppose that the fundamental group of its twofold branched cyclic cover is not left-orderable. If $\rho: \pi_{1}\left(S^{3} \backslash K\right) \rightarrow$ Homeo $_{+}\left(S^{1}\right)$ is a homomorphism such that $\rho\left(\mu^{2}\right)=1$ for some meridional class $\mu$ in $\pi_{1}\left(S^{3} \backslash K\right)$, then the image of $\rho$ is either trivial or isomorphic to $\mathbb{Z}_{2}$.

Here we make two remarks to compare Theorem 3.1 with [3, Theorem 6].

- The proof of [3, Theorem 6] naturally extends to the $n^{\text {th }}$ cyclic branched cover for arbitrary $n$. Since PSL $(2, \mathbb{R})$ is a subgroup of $\mathrm{Homeo}_{+}\left(S^{1}\right)$, the group of orientation preserving homeomorphisms of $S^{1}$, Theorem 3.1 is contained in [3, Theorem 6] in this sense.

- On the other hand, if we replace the central extension

$$
0 \longrightarrow \mathbb{Z} \longrightarrow \widetilde{\mathrm{SL}}(2, \mathbb{R}) \longrightarrow \mathrm{SL}(2, \mathbb{R}) \longrightarrow 1
$$

that we use in the proof of Theorem 3.1 by the extension [8]

$$
0 \longrightarrow \mathbb{Z} \longrightarrow \widetilde{\text { Homeo }}_{+}\left(S^{1}\right) \longrightarrow \text { Homeo }_{+}\left(S^{1}\right) \longrightarrow 1,
$$

we can achieve a proof of [3, Theorem 6].

Finally, in Section 4, we prove our main result in this paper.

Theorem 4.3 A $(p, q)$ two-bridge knot $K$ with $p \equiv 3 \bmod 4$ has only finitely many cyclic branched covers whose fundamental groups are not left-orderable.

Acknowledgements The author would like to thank Oliver Dasbach for drawing her attention to the topic of the current paper and his consistent encouragement and support throughout her graduate study. Also, the author would like to give thanks to Michel Boileau, Tye Lidman and Neal Stoltzfus for helpful conversation and suggestions. Finally, she gives many thanks to the referee for pointing out the similarities between Theorem 3.1 and [3, Theorem 6] and his or her many helpful comments. 


\section{The fundamental groups of cyclic branched covers}

Given a Seifert surface $F$, one can present the knot group $\pi_{1}\left(X_{K}\right)$ as an HNN extension of $\pi_{1}\left(S^{3} \backslash F\right)$ over the surface group $\pi_{1}(F)$, (the usual definition of the HNN extension requires $F$ to be incompressible, but we do not need it here). We then apply the Reidemeister-Schreier method to the presentation of $\pi_{1}\left(X_{K}\right)$ and obtain a presentation of $\pi_{1}\left(X_{K}^{(n)}\right)$, from which Lemma 2.1 follows.

More precisely, let $F$ be a Seifert surface of an oriented knot $K$. It has a regular neighborhood that is homeomorphic to $F \times[-1,1]$, where the positive direction is chosen so that the induced orientation on the boundary $\partial F$ is the same as the chosen orientation on the knot $K$.

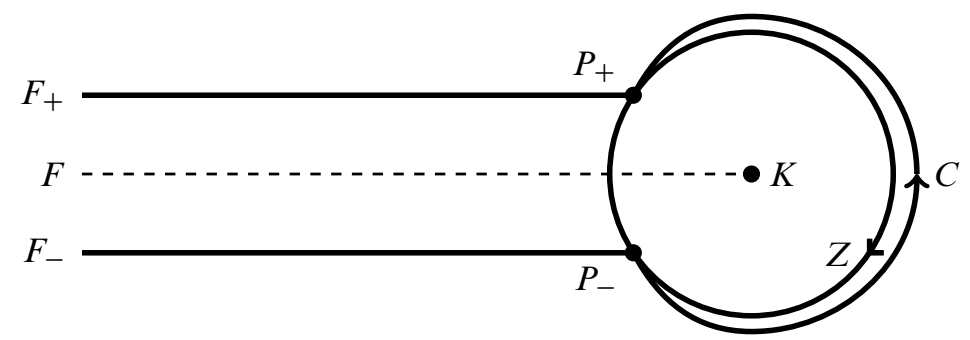

Figure 1: A cross-sectional view of a collar neighborhood of $F$ in the knot complement $X_{K}$, where $F_{ \pm}$represent $F \times \pm 1$, respectively. In addition, the point $P_{+}$(resp. $\left.P_{-}\right)$is the intersection point of the meridian $Z$ and $F_{+}$ (resp. $F_{-}$).

Suppose that the free groups $\pi_{1}\left(F_{-}, P_{-}\right)$and $\pi_{1}\left(F_{+}, P_{+}\right)$are generated by the elements $\left\{a_{i}^{-}\right\}_{i=1, \ldots, 2 g}$ and $\left\{a_{i}^{+}\right\}_{i=1, \ldots, 2 g}$ respectively, where $g$ is the genus of the Seifert surface $F$.

We denote by $\alpha_{i}^{-}$the image of $a_{i}^{-}$under the inclusion map

$$
\pi_{1}\left(F_{-}, P_{-}\right) \longrightarrow \pi_{1}\left(S^{3}-F, P_{-}\right)
$$

and denote by $\alpha_{i}^{+}$the image of $a_{i}^{+}$in $\pi_{1}\left(S^{3}-F, P_{-}\right)$under the composition map

$$
\pi_{1}\left(F_{+}, P_{+}\right) \longrightarrow \pi_{1}\left(S^{3}-F, P_{+}\right) \longrightarrow \pi_{1}\left(S^{3}-F, P_{-}\right),
$$

where the second map from $\pi_{1}\left(S^{3}-F, P_{+}\right)$to $\pi_{1}\left(S^{3}-F, P_{-}\right)$is the isomorphism induced by the $\operatorname{arc} C$ connecting $P_{-}$to $P_{+}$as depicted in Figure 1 . By the van Kampen theorem, we have

$$
\pi_{1}\left(X_{K}, P_{-}\right)=\pi_{1}\left(S^{3}-F, P_{-}\right) *\langle Z\rangle /\left\langle\left\langle Z \alpha_{i}^{+} Z^{-1}=\alpha_{i}^{-}, i=1, \ldots, 2 g\right\rangle\right\rangle .
$$


If the complement of the Seifert surface $F$ in $S^{3}$ is also a handlebody, which is always the case when $F$ is constructed through Seifert's algorithm, then the group $\pi_{1}\left(S^{3}-F, P_{-}\right)$is also free and we assume that

$$
\pi_{1}\left(S^{3}-F, P_{-}\right)=\left\langle x_{1}, \ldots, x_{2 g}\right\rangle .
$$

In this case, from (1), we obtain Lin's presentation for the knot group $\pi_{1}\left(X_{K}, P_{-}\right)$ [16, Lemma 2.1] as

$$
\pi_{1}\left(X, P_{-}\right)=\left\langle x_{1}, x_{2}, \ldots, x_{2 g-1}, x_{2 g}, Z: Z \alpha_{i}^{+} Z^{-1}=\alpha_{i}^{-}, i=1, \ldots, 2 g\right\rangle,
$$

where $\alpha_{i}^{ \pm}$are words in $x_{i}$ as described above.

Let $\tilde{X}_{K}^{(n)}$ be the $n^{\text {th }}$ cyclic cover of the knot complement $X_{K}$. Its fundamental group

$$
\pi_{1}\left(\tilde{X}_{K}^{(n)}\right) \cong \operatorname{Ker}\left(\pi_{1}\left(X_{K}\right) \longrightarrow \mathbb{Z}_{n}\right)
$$

is an index- $n$ subgroup of the knot group $\pi_{1}\left(X_{K}\right)$. Choose $\left\{Z^{i}\right\}_{i=0, \ldots, n-1}$ to be the representative from each coset. By applying the Reidemeister-Schreier method [17] to the presentation (2), we obtain a presentation of the group $\pi_{1}\left(\tilde{X}_{K}^{(n)}\right)$ with generators

$$
Z^{n} \quad \text { and } Z^{k} x_{1} Z^{-k}, \ldots, Z^{k} x_{2 g} Z^{-k} \text { for } k=0, \ldots, n-1
$$

and relations

$$
\begin{array}{ll}
Z^{k+1} \alpha_{i}^{+} Z^{-(k+1)}=Z^{k} \alpha_{i}^{-} Z^{-k} & \text { for } k=0, \ldots, n-2 \text { and } i=1, \ldots, 2 g \\
Z^{n} \cdot \alpha_{i}^{+} \cdot Z^{-n}=Z^{n-1} \alpha_{i}^{-} Z^{-(n-1)} & \text { for } i=1, \ldots, 2 g
\end{array}
$$

In the presentation above, $Z^{k} x_{i} Z^{-k}$ and $Z^{n}$ should be viewed as abstract symbols rather than products of $Z$ and $x_{i}$. Thus, words $Z^{k} \alpha_{i}^{+} Z^{-k}$ as in (3) are products of the generators $Z^{k} x_{i} Z^{-k}$ and the word $Z^{n} \cdot \alpha_{i}^{+} \cdot Z^{-n}$ in (4) is the product of $Z^{ \pm n}$ and $x_{i}$. The notation is chosen to emphasize the fact that the isomorphism between the presented group and the subgroup $\operatorname{Ker}\left(\pi_{1}\left(X_{K}\right) \rightarrow \mathbb{Z}_{n}\right)$ is given by sending the abstract symbol $Z^{k} x_{i} Z^{-k}$ in the presentation to the element $Z^{k} x_{i} Z^{-k}$ of the knot group $\pi_{1}\left(X_{K}\right)$ for $k=0, \ldots, n-1$ and $i=1, \ldots, 2 g$.

Intuitively, this presentation can be understood as follows. The $n^{\text {th }}$ cyclic cover $\tilde{X}_{K}^{(n)}$ can be constructed by gluing $n$ copies of $S^{3}-F \times(-1,1)$ together. We denote each copy by $Y_{k}$. Let $F_{k}$ be the Seifert surface associated with $Y_{k}$ and $F_{k}^{ \pm}$be $F_{k} \times \pm 1$ on $\partial Y_{k}$ for $k=0, \ldots, n-1$. Then $Z^{k} x_{i} Z^{-k}$ are generator loops in $Y_{k}$ and each relation $Z^{k+1} \alpha_{i}^{+} Z^{-(k+1)}=Z^{k} \alpha_{i}^{-} Z^{-k}$ in (3) is due to the isomorphism between $\pi_{1}\left(F_{k}^{-}\right)$and $\pi_{1}\left(F_{k+1}^{+}\right)$. In addition, the relation (4) is from the identification between $F_{0}^{+}$and $F_{n-1}^{-}$. 
Now let's look at the fundamental group of the $n^{\text {th }}$ cyclic branched cover $X_{K}^{(n)}$. From the construction of $X_{K}^{(n)}$, we have the isomorphism

$$
\pi_{1}\left(X_{K}^{(n)}\right) \cong \operatorname{Ker}\left(\pi_{1}\left(X_{K}\right) \rightarrow \mathbb{Z}_{n}\right) /\left\langle\left\langle Z^{n}\right\rangle\right\rangle
$$

Therefore the group $\pi_{1}\left(X_{K}^{(n)}\right)$ inherits the presentation with generators

$$
Z^{k} x_{1} Z^{-k}, \ldots, Z^{k} x_{2 g} Z^{-k} \text { for } k=0, \cdots, n-1
$$

and relations

$$
\begin{array}{cc}
Z^{k+1} \alpha_{i}^{+} Z^{-(k+1)}=Z^{k} \alpha_{i}^{-} Z^{-k} & \text { for } k=0, \cdots, n-2 \text { and } i=1, \ldots, 2 g, \\
\alpha_{i}^{+}=Z^{n-1} \alpha_{i}^{-} Z^{-(n-1)} & \text { for } i=1, \ldots, 2 g
\end{array}
$$

Lemma 2.1 Given a knot $K$ in $S^{3}$, denote by $Z$ a meridional element in the knot group $\pi_{1}\left(X_{K}\right)$. Suppose that there exists a group homomorphism $\rho$ from $\pi_{1}\left(X_{K}\right)$ to a group $G$ and $\rho\left(Z^{n}\right)$ is in the center of $G$. Then $\rho$ induces a group homomorphism from $\pi_{1}\left(X_{K}^{(n)}\right)$ to $G$. In particular, if $\rho$ is nonabelian, then the induced homomorphism is nontrivial.

Proof Let $\left.\rho\right|_{\text {ker }}$ be the restriction of $\rho$ to the subgroup $\operatorname{Ker}\left(\pi_{1}\left(X_{K}\right) \rightarrow \mathbb{Z}_{n}\right)$. We are going to show that the assignment

$$
\left.Z^{k} x_{i} Z^{-k} \mapsto \rho\right|_{\text {ker }}\left(Z^{k} x_{i} Z^{-k}\right) \quad \text { for } i=1, \ldots, 2 g \text { and } k=0, \ldots, n-1
$$

also defines a homomorphism from $\pi_{1}\left(X_{K}^{(n)}\right)$ to $G$.

First of all, the relations in (3) which are the same as the relations in (5) automatically hold. It follows from (4) that

$$
\left.\left.\left.\rho\right|_{\operatorname{ker}}\left(Z^{n}\right) \cdot \rho\right|_{\operatorname{ker}}\left(\alpha_{i}^{+}\right) \cdot \rho\right|_{\operatorname{ker}}\left(Z^{-n}\right)=\left.\rho\right|_{\operatorname{ker}}\left(Z^{n-1} \alpha_{i}^{-} Z^{-(n-1)}\right) .
$$

Since by assumption $\left.\rho\right|_{\text {ker }}\left(Z^{n}\right)=\rho\left(Z^{n}\right)$ is in the center of $G$, we have

$$
\left.\rho\right|_{\operatorname{ker}}\left(\alpha_{i}^{+}\right)=\left.\left.\left.\rho\right|_{\operatorname{ker}}\left(Z^{n}\right) \cdot \rho\right|_{\operatorname{ker}}\left(\alpha_{i}^{+}\right) \cdot \rho\right|_{\operatorname{ker}}\left(Z^{-n}\right)=\left.\rho\right|_{\operatorname{ker}}\left(Z^{n-1} \alpha_{i}^{-} Z^{-(n-1)}\right) .
$$

That is, the relations in (6) hold as well.

In addition, if $\rho$ is a nonabelian homomorphism, then as the commutator subgroup $\left[\pi_{1}\left(X_{K}\right), \pi_{1}\left(X_{K}\right)\right]$ is the normal subgroup generated by $\left\{x_{1}, \ldots, x_{2}\right\}$, we have that $\rho\left(x_{i}\right)$ is not equal to the identity in $G$ for some $i$. Therefore, the induced homomorphism from $\pi_{1}\left(X_{K}^{(n)}\right)$ to $G$ is nontrivial. 


\section{The left-orderability of the fundamental group $\pi_{1}\left(X_{K}^{(n)}\right)$}

We finish the proof of Theorem 3.1 in this section.

Theorem 3.1 Given any prime knot $K$ in $S^{3}$, denote by $Z$ a meridional element of $\pi_{1}\left(X_{K}\right)$. If there exists a nonabelian representation $\pi_{1}\left(X_{K}\right)$ to $\operatorname{SL}(2, \mathbb{R})$ such that $Z^{n}$ is sent to $\pm I$ then the fundamental group $\pi_{1}\left(X_{K}^{(n)}\right)$ is left-orderable.

We will make use of the following criterion due to Boyer, Rolfsen and Wiest.

Theorem 3.2 [4] Let $M$ be a compact, orientable, irreducible 3-manifold. Then $\pi_{1}(M)$ is left-orderable, if there exists a nontrivial homomorphism from $\pi_{1}(M)$ to a left-orderable group.

Note that the group $\operatorname{SL}(2, \mathbb{R})$ itself is not left-orderable, but its universal covering group, denoted by $\widetilde{\mathrm{SL}}(2, \mathbb{R})$, is left-orderable [1]. Let $E$ be the covering map from $\widetilde{\mathrm{SL}}(2, \mathbb{R})$ to $\operatorname{SL}(2, \mathbb{R})$. Since $\widetilde{\mathrm{SL}}(2, \mathbb{R})$ and $\operatorname{SL}(2, \mathbb{R})$ are both connected, we have

$$
\mathcal{Z}(\widetilde{\mathrm{SL}}(2, \mathbb{R}))=E^{-1}(\mathcal{Z}(\operatorname{SL}(2, \mathbb{R}))),
$$

where $\mathcal{Z}(\widetilde{\mathrm{SL}}(2, \mathbb{R}))$ and $\mathcal{Z}(\operatorname{SL}(2, \mathbb{R}))$ are the centers of the Lie groups $\widetilde{\mathrm{SL}}(2, \mathbb{R})$ and $\operatorname{SL}(2, \mathbb{R})$ respectively $\left[11\right.$, page 336]. Therefore, $\mathcal{Z}(\widetilde{\mathrm{SL}}(2, \mathbb{R}))=E^{-1}(\{ \pm I\})$.

Lemma 3.3 Given any knot $K$ in $S^{3}$, let $Z$ be a meridional element in the knot group $\pi_{1}\left(X_{K}\right)$. Suppose that there exists a nonabelian $\operatorname{SL}(2, \mathbb{R})$ representation of $\pi_{1}\left(X_{K}\right)$ such that $Z^{n}$ is sent to $\pm I$. Then this representation induces a nontrivial $\widetilde{\mathrm{SL}}(2, \mathbb{R})$ representation of the fundamental group of the $n^{\text {th }}$ cyclic branched cover $\pi_{1}\left(X_{K}^{(n)}\right)$.

Proof The kernel of the covering map $\operatorname{Ker}(E)$ is isomorphic to $\pi_{1}(\operatorname{SL}(2, \mathbb{R})) \cong \mathbb{Z}$ and we have the central extension

$$
0 \longrightarrow \mathbb{Z} \longrightarrow \widetilde{\mathrm{SL}}(2, \mathbb{R}) \longrightarrow \mathrm{SL}(2, \mathbb{R}) \longrightarrow I .
$$

Suppose that $\rho$ is a representation of $\pi_{1}\left(X_{K}\right)$ into $\operatorname{SL}(2, \mathbb{R})$. Then the pullback

$$
\widetilde{\mathrm{SL}}(2, \mathbb{R}) \times_{\mathrm{SL}(2, \mathbb{R})} \pi_{1}\left(X_{K}\right)=\left\{(M, x) \in \widetilde{\mathrm{SL}}(2, \mathbb{R}) \times \pi_{1}\left(X_{K}\right): E(M)=\rho(x)\right\},
$$

is a central extension of $\pi_{1}(X)$ by $\mathbb{Z}$. On the other hand,

$$
H^{2}\left(\pi_{1}\left(X_{K}\right), \mathbb{Z}\right) \cong H^{2}\left(X_{K}, \mathbb{Z}\right)=0,
$$

so every central extension of $\pi_{1}\left(X_{k}\right)$ by $\mathbb{Z}$ splits. Hence, $\rho$ can be lifted to a representation into $\widetilde{S L}(2, \mathbb{R})$. That is, the composition of a splitting map with the projection from $\widetilde{\mathrm{SL}}(2, \mathbb{R}) \times_{\mathrm{SL}(2, \mathbb{R})} \pi_{1}\left(X_{K}\right)$ to $\widetilde{\mathrm{SL}}(2, \mathbb{R})$ is a lifting of $\rho$ [27]. 
Now assume that the representation $\rho$ of the knot group $\pi_{1}\left(X_{K}\right)$ satisfies the property $\rho\left(Z^{n}\right)= \pm I$. We denote by $\tilde{\rho}$ a lifting of $\rho$. Since $\rho\left(Z^{n}\right)= \pm I$, we see that $\tilde{\rho}\left(Z^{n}\right)$ is inside $E^{-1}( \pm I)$, which is equal to $\mathcal{Z}(\widetilde{\mathrm{SL}}(2, \mathbb{R}))$, the center of $\widetilde{\mathrm{SL}}(2, \mathbb{R})$.

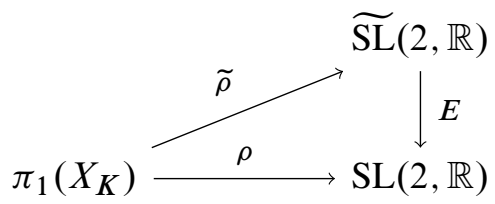

In addition, if $\rho$ is a nonabelian representation, then $\tilde{\rho}$ is nonabelian. By Lemma 2.1, the representation $\tilde{\rho}$ induces a nontrivial $\widetilde{\mathrm{SL}}(2, \mathbb{R})$ representation of $\pi_{1}\left(X_{K}^{(n)}\right)$.

Proof of Theorem 3.1 Let $\rho$ be a nonabelian $\operatorname{SL}(2, \mathbb{R})$-representation of the knot group $\pi_{1}\left(X_{K}\right)$, with $\rho\left(Z^{n}\right)= \pm I$. By Lemma 3.3, this representation induces a nontrivial $\widetilde{\mathrm{SL}}(2, \mathbb{R})$-representation of the group $\pi_{1}\left(X_{K}^{(n)}\right)$.

The group $\widetilde{\mathrm{SL}}(2, \mathbb{R})$ can be embedded inside the group of order-preserving homeomorphisms of $\mathbb{R}$, so it is left-orderable [1]. Moreover, the $n^{\text {th }}$ cyclic branched cover $X_{K}^{(n)}$ is irreducible if $K$ is a prime knot [22]. Thus, Theorem 3.1 follows from Theorem 3.2.

\section{Application to $(p, q)$ two-bridge knots with $p \equiv 3 \bmod 4$}

In this section we apply Theorem 3.1 to $(p, q)$ two-bridge knots with $p=3 \bmod 4$. We show that given any two-bridge knot of this type, the fundamental group of the $n^{\text {th }}$ cyclic branched cover is left-orderable if $n$ is sufficiently large.

Let $K$ be a $(p, q)$ two-bridge knot. From the Schubert normal form [14, page 21], the knot group has a presentation of the form

$$
\pi_{1}\left(X_{K}\right)=\langle x, y: w x=y w\rangle,
$$

where $w=\left(x^{\epsilon_{1}} y^{\epsilon_{2}}\right) \cdots\left(x^{\epsilon_{p-2}} y^{\epsilon_{p-1}}\right)$ and $\epsilon_{i}= \pm 1$.

Let $\rho: \pi_{1}\left(X_{K}\right) \rightarrow \operatorname{SL}(2, \mathbb{C})$ be a nonabelian representation of the knot group into $\operatorname{SL}(2, \mathbb{C})$. Up to conjugation, we can assume that

$$
\rho(x)=\left(\begin{array}{cc}
m & 1 \\
0 & m^{-1}
\end{array}\right), \quad \rho(y)=\left(\begin{array}{cc}
m & 0 \\
s & m^{-1}
\end{array}\right) .
$$

Hence $\rho(w)=\rho(x)^{\epsilon_{1}} \rho(y)^{\epsilon_{2}} \cdots \rho(x)^{\epsilon_{p-2}} \rho(y)^{\epsilon_{p-1}}$ is a matrix with entries in $\mathbb{Z}\left[m^{ \pm 1}, s\right]$; we write

$$
\rho(w)=\left(\begin{array}{ll}
w_{11} & w_{12} \\
w_{21} & w_{22}
\end{array}\right), \quad w_{i j} \in \mathbb{Z}\left[m^{ \pm 1}, s\right] .
$$


From the group relation $w x=y w$, we have

$$
\left(\begin{array}{ll}
w_{11} & w_{12} \\
w_{21} & w_{22}
\end{array}\right)\left(\begin{array}{cc}
m & 1 \\
0 & m^{-1}
\end{array}\right)=\left(\begin{array}{cc}
m & 0 \\
s & m^{-1}
\end{array}\right)\left(\begin{array}{ll}
w_{11} & w_{12} \\
w_{21} & w_{22}
\end{array}\right)
$$

This is equivalent to

$$
\left(\begin{array}{cc}
0 & w_{11}+\left(m^{-1}-m\right) w_{12} \\
\left(m-m^{-1}\right) w_{21}-s w_{11} & w_{21}-s w_{12}
\end{array}\right)=0,
$$

so $s$ and $m$ must satisfy the equation

$$
w_{11}+\left(m^{-1}-m\right) w_{12}=0 .
$$

The above equation is also a sufficient condition for $\rho$ to define a representation:

Proposition 4.1 [24, Theorem 1] The assignment of $x$ and $y$ as in (7) defines a nonabelian $\mathrm{SL}(2, \mathbb{C})$ representation of the knot group

$$
\pi_{1}\left(X_{K}\right)=\langle x, y: w x=y w\rangle
$$

if and only if

$$
\varphi(m, s) \triangleq w_{11}+\left(m^{-1}-m\right) w_{12}=0 .
$$

We need to make use of several properties of Riley's polynomial $\varphi(m, s)$. All of these properties are either proven or claimed in Riley's paper [24]. For readers' convenience, we organize them and provide a proof in the following lemma.

Lemma 4.2 (cf [24]) The polynomial $\varphi(m, s)$ in $\mathbb{Z}\left[m^{ \pm 1}, s\right]$ satisfies the following:

(1) As a polynomial in $s$ with coefficients in $\mathbb{Z}\left[m^{ \pm 1}\right], \varphi(m, s)$ has $s$-degree equal to $(p-1) / 2$, with the leading coefficient \pm 1 .

(2) $\varphi(1,0) \neq 0$.

(3) $\varphi(m, s)$ does not have repeated factors.

(4) $\varphi(m, s)=\varphi\left(m^{-1}, s\right)$ and thus $\varphi(m, s)=f\left(m+m^{-1}, s\right)$, where $f$ is a twovariable polynomial with coefficients in $\mathbb{Z}$.

Proof (1) Since we assign

$$
\rho(x)=\left(\begin{array}{cc}
m & 1 \\
0 & m^{-1}
\end{array}\right), \quad \rho(y)=\left(\begin{array}{cc}
m & 0 \\
s & m^{-1}
\end{array}\right),
$$


a direct computation gives

$$
\begin{aligned}
& \rho(x y)=\left(\begin{array}{cc}
m^{2}+s & m^{-1} \\
m^{-1} s & m^{-2}
\end{array}\right), \quad \rho\left(x^{-1} y\right)=\left(\begin{array}{cc}
1-s & -m^{-1} \\
m s & 1
\end{array}\right), \\
& \rho\left(x y^{-1}\right)=\left(\begin{array}{cc}
1-s & m \\
-m^{-1} s & 1
\end{array}\right), \quad \rho\left(x^{-1} y^{-1}\right)=\left(\begin{array}{cc}
m^{-2}+s & -m \\
-m s & m^{2}
\end{array}\right) \text {. }
\end{aligned}
$$

Say a matrix $A$ in $M_{2}\left(\mathbb{Z}\left[m^{ \pm 1}, s\right]\right)$ has $s$-degree equal to $n$ if

$$
A=\left(\begin{array}{cc} 
\pm s^{n}+f_{11}(m, s) & f_{12}(m, s) \\
f_{21}(m, s) & f_{22}(m, s)
\end{array}\right),
$$

where the $s$-degrees of $f_{11}, f_{12}$ and $f_{22}$ are strictly less than $n$ and the $s$-degree of $f_{21}$ is less than or equal to $n$. Hence the matrices $\rho(x y), \rho\left(x^{-1} y\right), \rho\left(x y^{-1}\right)$ and $\rho\left(x^{-1} y^{-1}\right)$ all have $s$-degrees equal to 1 . Moreover, the product of an $s$-degree $n$ matrix and an $s$-degree $m$ matrix is an $s$-degree $m+n$ matrix. Since

$$
w=\left(x^{\epsilon_{1}} y^{\epsilon_{2}}\right) \cdots\left(x^{\epsilon_{p-2}} y^{\epsilon_{p-1}}\right), \quad \epsilon_{i}= \pm 1,
$$

the matrix

$$
\rho(w)=\left(\begin{array}{ll}
w_{11} & w_{12} \\
w_{21} & w_{22}
\end{array}\right)
$$

is a product of $(p-1) / 2 s$-degree 1 matrices. Therefore the matrix $\rho(w)$ has $s-$ degree equal to $(p-1) / 2$. That is, the entry $w_{11}$ has $\pm s(p-1) / 2$ as the leading term and the $s$-degree of $w_{12}$ is strictly less than $(p-1) / 2$. As a result, $\varphi(m, s)=$ $w_{11}+\left(m^{-1}-m\right) w_{12}$ has leading term equal to $\pm s(p-1) / 2$.

(2) Since $m=1$ and $s=0$, we have

$$
\rho(x)=\left(\begin{array}{ll}
1 & 1 \\
0 & 1
\end{array}\right), \quad \rho(y)=\left(\begin{array}{ll}
1 & 0 \\
0 & 1
\end{array}\right) .
$$

This assignment can not define a representation of the knot group

$$
\pi_{1}\left(X_{K}\right)=\langle x, y: w x=y w\rangle
$$

because the matrices $\rho(x)=\left(\begin{array}{ll}1 & 1 \\ 0 & 1\end{array}\right)$ and $\rho(y)=\left(\begin{array}{ll}1 & 0 \\ 0 & 1\end{array}\right)$ are not conjugate to each other. Therefore $\varphi(1,0) \neq 0$ by Proposition 4.1 .

(3) Let $\Delta_{K}(t)$ be the Alexander polynomial of the knot $K$. It is shown in [18, Proposition 1.1, Theorem 1.2] that any knot group has $\left(\left|\Delta_{K}(-1)\right|-1\right) / 2$ irreducible $\mathrm{SL}(2, \mathbb{C})$ metabelian representations up to conjugation (see also $[2 ; 16]$ ) and that these metabelian representations send meridional elements to matrices of eigenvalues $\pm i$. For a $(p, q)$ two-bridge knot, $p$ equals $\left|\Delta_{K}(-1)\right|$. This implies that the degree- $(p-1) / 2$ 
polynomial equation $\varphi(i, s)=0$ has $(p-1) / 2$ distinguished roots. Therefore $\varphi(i, s)$ does not have repeated factors and neither does $\varphi(m, s)$.

Note that we can also use the fact that $\varphi(1, s)$ does not have any repeated factors to prove that $\varphi(m, s)$ has no repeated factors [23, Theorem 3].

(4) Assume that the assignment

$$
\rho(x)=\left(\begin{array}{cc}
m & 1 \\
0 & m^{-1}
\end{array}\right), \quad \rho(y)=\left(\begin{array}{cc}
m & 0 \\
s & m^{-1}
\end{array}\right)
$$

defines a representation of the knot group

$$
\pi_{1}\left(X_{K}\right)=\langle x, y: w x=y w\rangle .
$$

Then

$$
\begin{aligned}
& \rho^{\prime}(x)=P\left(\begin{array}{cc}
m & 1 \\
0 & m^{-1}
\end{array}\right) P^{-1}=\left(\begin{array}{cc}
m^{-1} & 1 \\
0 & m
\end{array}\right), \\
& \rho^{\prime}(y)=P\left(\begin{array}{cc}
m & 0 \\
s & m^{-1}
\end{array}\right) P^{-1}=\left(\begin{array}{cc}
m^{-1} & 0 \\
s & m
\end{array}\right),
\end{aligned}
$$

also defines a representation, where

$$
P=\left(\begin{array}{cc}
1 & \left(m^{-1}-m\right) / s \\
m-m^{-1} & 1
\end{array}\right) .
$$

The matrix $P$ is well-defined and invertible whenever $(m, s)$ is not in the finite set

$$
S \triangleq\{(m, s): s=0, \varphi(m, s)=0\} \cup\left\{(m, s): s=-\left(m-m^{-1}\right)^{2}, \varphi(m, s)=0\right\} .
$$

The set $S$ is finite because neither $\varphi(m, 0)$ nor $\varphi\left(m,-\left(m-m^{-1}\right)^{2}\right)$ is a zero polynomial. Otherwise, $(1,0)$ would be a solution for $\varphi(m, s)$, which contradicts part (2).

Denote by $V(g)$ the solution set of a polynomial $g$. As we described above,

$$
V(\varphi(m, s))-S \subset V(\psi(m, s)),
$$

where $\psi(m, s)=\varphi\left(m^{-1}, s\right)$. Points in $S$ are not isolated, since they are embedded inside the algebraic curve $V(\varphi(m, s))$. By continuity, we have

$$
V(\varphi(m, s)) \subset V(\psi(m, s)) .
$$

By part (3), neither of $\varphi(m, s)$ nor $\psi(m, s)$ have repeated factors, so the ideal $\langle\psi(m, s)\rangle$ is contained inside the ideal $\langle\varphi(m, s)\rangle$ in $\mathbb{Z}\left[m^{ \pm 1}, s\right]$. On the other hand, both $\varphi(m, s)$ and $\psi(m, s)$ have the same leading term, which is either $s^{(p-1) / 2}$ or $-s^{(p-1) / 2}$, so $\varphi(m, s)=\psi(m, s)=\varphi\left(m^{-1}, s\right)$. 
Now we are ready to prove the main result.

Theorem $4.3 A(p, q)$ two-bridge knot $K$ with $p \equiv 3 \bmod 4$ has only finitely many cyclic branched covers whose fundamental groups are not left-orderable.

Proof We are going to show that for sufficiently large $n$, the group

$$
\pi_{1}\left(X_{K}\right)=\langle x, y: w x=y w\rangle
$$

has a nonabelian $\operatorname{SL}(2, \mathbb{R})$-representation with $x^{n}$ sent to $-I$.

As before, we set

$$
\rho(x)=\left(\begin{array}{cc}
m & 1 \\
0 & m^{-1}
\end{array}\right), \quad \rho(y)=\left(\begin{array}{cc}
m & 0 \\
s & m^{-1}
\end{array}\right) .
$$

Let $m=e^{i \theta}$. Since $p=3 \bmod 4$, by Lemma 4.2 , we have that $\varphi\left(e^{i \theta}, s\right)$ is an odddegree real polynomial in $s$. So for any given $\theta$, the equation $\varphi\left(e^{i \theta}, s\right)=0$ has at least one real solution for $s$. We assume that $s_{0}$ is a real solution of the equation $\varphi(1, s)=0$. It is known that the polynomial $\varphi(1, s)$ does not have repeated factors [23, Theorem 3]. Hence $\left.\varphi_{s}\left(e^{i \theta}, s\right)\right|_{\theta=0, s=s_{0}} \neq 0$ and locally there exists a real function $s(\theta)$ such that $\varphi\left(e^{i \theta}, s(\theta)\right)=0$ and $s(0)=s_{0}$.

Consider the one-parameter family of nonabelian representations

$$
\rho\{\theta\}(x)=\left(\begin{array}{cc}
e^{i \theta} & 1 \\
0 & e^{-i \theta}
\end{array}\right), \quad \rho\{\theta\}(y)=\left(\begin{array}{cc}
e^{i \theta} & 0 \\
s(\theta) & e^{-i \theta}
\end{array}\right) .
$$

As $\theta \neq 0$, the representations $\rho\{\theta\}$ can be diagonalized to the following forms, which we still denote by $\rho\{\theta\}$ :

$$
\rho\{\theta\}(x)=\left(\begin{array}{cc}
e^{i \theta} & 0 \\
0 & e^{-i \theta}
\end{array}\right), \quad \rho\{\theta\}(y)=\left(\begin{array}{cc}
e^{i \theta}-\frac{s(\theta)}{2 \sin (\theta)} i & -1+\frac{s(\theta)}{4 \sin ^{2}(\theta)} \\
s(\theta) & e^{-i \theta}+\frac{s(\theta)}{2 \sin (\theta)} i
\end{array}\right) .
$$

According to [15, page 786], this representation can be conjugated to an $\operatorname{SL}(2, \mathbb{R})-$ representation if and only if either

$$
s(\theta)<0 \quad \text { or } \quad s(\theta)>4 \sin ^{2}(\theta) .
$$

We can verify this by a direction computation. In fact, when $s<0$ or $s>4 \sin ^{2}(\theta)$, the representation $\rho\{\theta\}$ is conjugate to an $\mathrm{SU}(1,1)$-representation by the matrix

$$
\left(\begin{array}{cc}
\sqrt{\frac{1}{\sqrt{t}}+t} & t \\
\sqrt{t} & \sqrt{\sqrt{t}+t^{2}}
\end{array}\right) \quad \text { where } t=\frac{1}{4 \sin ^{2}(\theta)}-\frac{1}{s} \text { is positive, }
$$


and $\operatorname{SU}(1,1)$ is conjugate to $\operatorname{SL}(2, \mathbb{R})$ via the matrix $\left(\begin{array}{cc}1 & -i \\ 1 & i\end{array}\right)$ in $\operatorname{GL}(2, \mathbb{C})$.

On the other hand,

$$
\lim _{\theta \rightarrow 0} s(\theta)=s_{0},
$$

where $s_{0}$ is not equal to 0 by Lemma 4.2(2). Hence, when $\theta$ is small enough, either $s(\theta)<0$ or $s(\theta)>4 \sin ^{2}(\theta)$. Now let $\theta=\pi / n$. For sufficiently large $n$, the nonabelian representation $\rho\{\theta\}$ as in (10) satisfies $\rho\{\theta\}(x)^{n}=-I$ and conjugates to an $\operatorname{SL}(2, \mathbb{R})$ representation. Therefore, by Theorem 3.1, the conclusion follows.

Example 4.4 Consider the two bridge knot $(7,4)$, which is listed as $5_{2}$ in Rolfsen's table. The fundamental group $\pi_{1}\left(X_{5_{2}}\right)$ has a presentation

$$
\pi_{1}\left(X_{5_{2}}\right)=\langle x, y: w x=y w\rangle,
$$

where $w=x y x^{-1} y^{-1} x y$.

From this presentation, we can compute the polynomial

$$
\begin{aligned}
\varphi(m, s)=s^{3}+\left(2 \left(m^{2}+\right.\right. & \left.\left.m^{-2}\right)-3\right) s^{2} \\
& +\left(\left(m^{4}+m^{-4}\right)-3\left(m^{2}+m^{-2}\right)+6\right) s+2\left(m^{2}+m^{-2}\right)-3 .
\end{aligned}
$$

as defined in (9), and

$$
\varphi\left(e^{i \theta}, s\right)=s^{3}+(4 \cos (2 \theta)-3) s^{2}+(2 \cos (4 \theta)-6 \cos (2 \theta)+6) s+4 \cos (2 \theta)-3,
$$

which is a real polynomial in $s$ with degree 3 . Hence, we can find a closed formula for $s(\theta)$ such that $\varphi\left(e^{i \theta}, s(\theta)\right)=0$. Figure 2 is the graph of the solution $s(\theta)$ on the interval $\theta \in[0,1]$.

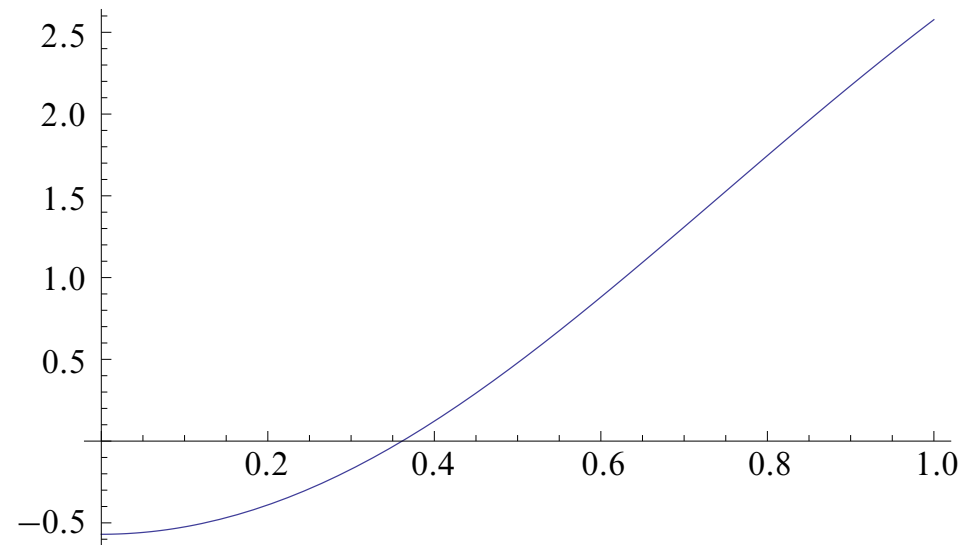

Figure 2 
In particular, when $n=9$, we have that $\frac{\pi}{9} \approx 0.349$ and $s\left(\frac{\pi}{9}\right) \approx-0.03667$. The group $\pi_{1}\left(X_{5_{2}}^{(n)}\right)$ is left-orderable when $n \geq 9$. For cyclic branched covers $X_{5_{2}}^{(n)}$ with $n<9$, the other known cases are $n=2,3$ [7] and $n=4$ [9], none of which has a left-orderable fundamental group.

\section{References}

[1] G M Bergman, Right orderable groups that are not locally indicable, Pacific J. Math. 147 (1991) 243-248 MR1084707

[2] H U Boden, S Friedl, Metabelian $\operatorname{SL}(n, \mathbb{C})$ representations of knot groups, Pacific J. Math. 238 (2008) 7-25 MR2443505

[3] S Boyer, CM Gordon, L Watson, On L-spaces and left-orderable fundamental groups, Math. Ann. 356 (2013) 1213-1245 MR3072799

[4] S Boyer, D Rolfsen, B Wiest, Orderable 3-manifold groups, Ann. Inst. Fourier (Grenoble) 55 (2005) 243-288 MR2141698

[5] D Calegari, N M Dunfield, Laminations and groups of homeomorphisms of the circle, Invent. Math. 152 (2003) 149-204 MR1965363

[6] A Clay, T Lidman, L Watson, Graph manifolds, left-orderability and amalgamation, Algebr. Geom. Topol. 13 (2013) 2347-2368 MR3073920

[7] M K Dąbkowski, J H Przytycki, A A Togha, Non-left-orderable 3-manifold groups, Canad. Math. Bull. 48 (2005) 32-40 MR2118761

[8] É Ghys, Groups acting on the circle, Enseign. Math. 47 (2001) 329-407 MR1876932

[9] C Gordon, T Lidman, Taut foliations, left-orderability, and cyclic branched covers, Acta Math. Vietnam. 39 (2014) 599-635 MR3292587

[10] J E Greene, Alternating links and left-orderability arXiv:1107.5232

[11] J Hilgert, K-H Neeb, Structure and geometry of Lie groups, Springer, New York (2012)

[12] J Howie, H Short, The band-sum problem, J. London Math. Soc. 31 (1985) 571-576 MR812788

[13] T Ito, Non-left-orderable double branched coverings, Algebr. Geom. Topol. 13 (2013) 1937-1965

[14] A Kawauchi, Survey on knot theory, Birkhäuser, Basel (1996)

[15] V T Khoi, A cut-and-paste method for computing the Seifert volumes, Math. Ann. 326 (2003) 759-801 MR2003451

[16] X S Lin, Representations of knot groups and twisted Alexander polynomials, Acta Math. Sin. (Engl. Ser.) 17 (2001) 361-380 MR1852950

[17] R C Lyndon, PE Schupp, Combinatorial group theory, Springer, Berlin (2001) MR1812024 
[18] F Nagasato, Finiteness of a section of the $\operatorname{SL}(2, \mathbb{C})$-character variety of the knot group, Kobe J. Math. 24 (2007) 125-136 MR2488756

[19] P Ozsváth, Z Szabó, On knot Floer homology and lens space surgeries, Topology 44 (2005) 1281-1300 MR2168576

[20] P Ozsváth, Z Szabó, On the Heegaard Floer homology of branched double-covers, Adv. Math. 194 (2005) 1-33 MR2141852

[21] T Peters, On L-spaces and non left-orderable 3-manifold groups arXiv:0903.4495

[22] S P Plotnick, Finite group actions and nonseparating 2-spheres, Proc. Amer. Math. Soc. 90 (1984) 430-432 MR728363

[23] R Riley, Parabolic representations of knot groups, I, Proc. London Math. Soc. 24 (1972) 217-242 MR0300267

[24] R Riley, Nonabelian representations of 2-bridge knot groups, Quart. J. Math. Oxford Ser. 35 (1984) 191-208 MR745421

[25] M Teragaito, Fourfold cyclic branched covers of genus one two-bridge knots are L-spaces, Bol. Soc. Mat. Mex. 20 (2014) 391-403 MR3264624

[26] A Tran, On left-orderablility and cyclic branched coverings to appear in J. Math. Soc. Japan

[27] C A Weibel, An introduction to homological algebra, Cambridge Studies Adv. Math. 38, Cambridge Univ. Press, Cambridge (1994) MR1269324

Department of Mathematics, Louisiana State University

Baton Rouge, LA 70803, USA

yhu4@math. Isu.edu

http: //www.math. Isu.edu/ yhu4

Received: 3 February 2014 Revised: 25 June 2014 
